\title{
Rationales for Establishing a Radiation Oncology Center in Fiji: Beyond Cost and Benefit Analysis
}

\author{
Chang Bae Chun \\ Department of Operation and Support, Korea Foundation for International Healthcare, Seoul, South Korea
}

\section{Email address:}

cbchun $a$ kofih.org

\section{To cite this article:}

Chang Bae Chun. Rationales for Establishing a Radiation Oncology Center in Fiji: Beyond Cost and Benefit Analysis. International Journal of Health Economics and Policy. Vol. 3, No. 2, 2018, pp. 20-27. doi: 10.11648/j.hep.20180302.12

Received: June 25, 2018; Accepted: July 13, 2018; Published: August 4, 2018

\begin{abstract}
In Fiji, cancers are the third leading cause of death behind cardiovascular diseases and diabetes. The cancer incidence is to increase rapidly, but cancer patients are not able to obtain treatment within the country. Instead they have to go abroad because radiotherapy services are not available in Fiji. To overcome the limited accessibility of cancer treatment, Fiji's government developed the National Cancer Control Plan and established a National Oncology Committee in 2014 to promote the establishment of a radiation oncology center. In spite of the government's willingness and much research, the implementation of the roadmap to build the oncology center has been delayed. To help the process avoid derailment, this study suggests rationales that go beyond cost-benefit analysis and other validations given in academic research. The three simple but strong rationales are suggested. Health sovereignty makes it the government's obligation to promote the health of the entire population. Governments should ensure the health of people by guaranteeing basic and necessary health services like cancer treatment. Universal Health Coverage (UHC) is an important goal for both developed and developing countries. Under UHC, everyone should be able to access the health services they need without financial hardship. This underscores the fact that UHC without cancer treatment is not possible. The development of a cancer treatment hub in the South Pacific region can be promoted as an important motivation for the establishment of a radiation oncology centre. Many countries in this region are too small to achieve economies of scale in their health sectors. Fiji has the potential to become a cancer treatment hub by establishing and operating a radiation therapy center for the populations of both Fiji and other neighboring countries.
\end{abstract}

Keywords: Radiation Oncology, Cost-Benefit Analysis, Health Sovereignty, Universal Health Coverage, Cancer Treatment Hub, Fiji

\section{Introduction}

Like many countries, the Republic of Fiji is not exceptional in experiencing an increased incidence of noncommunicable diseases (NCD), including cancer. According to Ministry of Health and Medical Services (MoHMS) 2014 data, the major burden of disease in Fiji is from NCDs. Among NCDs, cancer is the third leading cause of death behind cardiovascular diseases and diabetes [1]. According to the International Agency for Research on Cancer (IARC) GLOBOCAN projected, cancer burden in Fiji is expected to increase to 1437 cases per annum by 2025 [2], making NCDrelated diseases responsible for $80 \%$ of all deaths in Fiji [3]. The age standardized mortality rate from the top five cancers is $65.4 / 100,000$ in men and $104.3 / 100,000$ in women [4]. To cope with this problem, Fiji's "National Strategic Plan 2016-
2020" and National Cancer Control Strategic Plan include NCD as general objective, aiming a 22 percent decrease in premature mortality from NCDs by 2020 [5].

In this situation of rising numbers of cancer patients, accessibility to efficient cancer treatment is critically important, not to mention the need for cancer prevention programmes. Radiotherapy plays a key role in cancer treatment, either alone or in combination with the other major cancer treatment modalities like surgery and chemotherapy [2]. Nevertheless, radiotherapy treatment is not available to Fijian patients because currently no facility exists within the country. Patients have two choices: to go abroad to receive treatment or stay home. Patients who can afford to pay travel costs and accommodation for themselves and family can get permission from the selection committee to go to foreign countries (mainly India, New Zealand, and Australia), while 
other patients have to give up treatment. As a result, patients' conditions worsen by delaying timely treatment [4].

The public health system in Fiji is relatively good compared to that of other countries. Patients requiring inpatient and outpatient services can access medical facilities without paying out-of-pocket. However, cancer treatment services are different. Faced with the dilemma of having such an important gap in access to cancer treatment, the Fijian government started preparations for the establishment of a radiation therapy center in 2014. The Government and stakeholders have focused on satisfying cancer patients' needs and conducted academic research to gauge feasibility. A major task to pave the way for a radiation oncology center in Fiji was to gain national consensus effectively and efficiently. This study is based on the research and a situation analysis. It aims to contribute to reaching collective consensus and accelerating the provision of national cancer treatment services by presenting strong rationales for the establishment of a radiotherapy center.

\section{Research Method}

This study method on the establishment of a radiation oncology center in Fiji is mainly based on a literature review of previously published reports and documents. An analysis of the literature can provide information on the problem of cancer treatment, to cast light on government policy with regard to radiation therapy and to supply possible solutions.
Based on the literature review, the study also conducted interviews with stakeholders to check the findings and policy options suggested in the literature analysis. The interviews focusing on major stakeholders can be used as a means to understand the background of cancer polices in Fiji. In addition, the interviews with experts who have been involved in Fiji's oncology project provided valuable insights, making it possible to suggest rationales in support of establishing an oncology center, which have not been discussed in the previous studies. The study also used case analysis to look at other countries' oncology services. This case study will contribute to the development and sustainability of cancer services in Fiji by learning from foreign countries' experiences.

Based on these research methods the conceptual framework for this article can be depicted as in Figure 1 $<$ figure 1>. Situational analysis initially analyzed and followed up with literature reviews discussed in the next section. Based on previous studies, this study suggests rationales that support the necessity of establishing radiation oncology centers. Collating these rationales is designed to provide stakeholders with theoretical knowledge to strengthen the case for the establishment of an oncology center in Fiji. The final section discusses the outcomes and lessons learned from this study that can be used to influence policy makers and to contribute to the development of cancer treatment in Fiji.

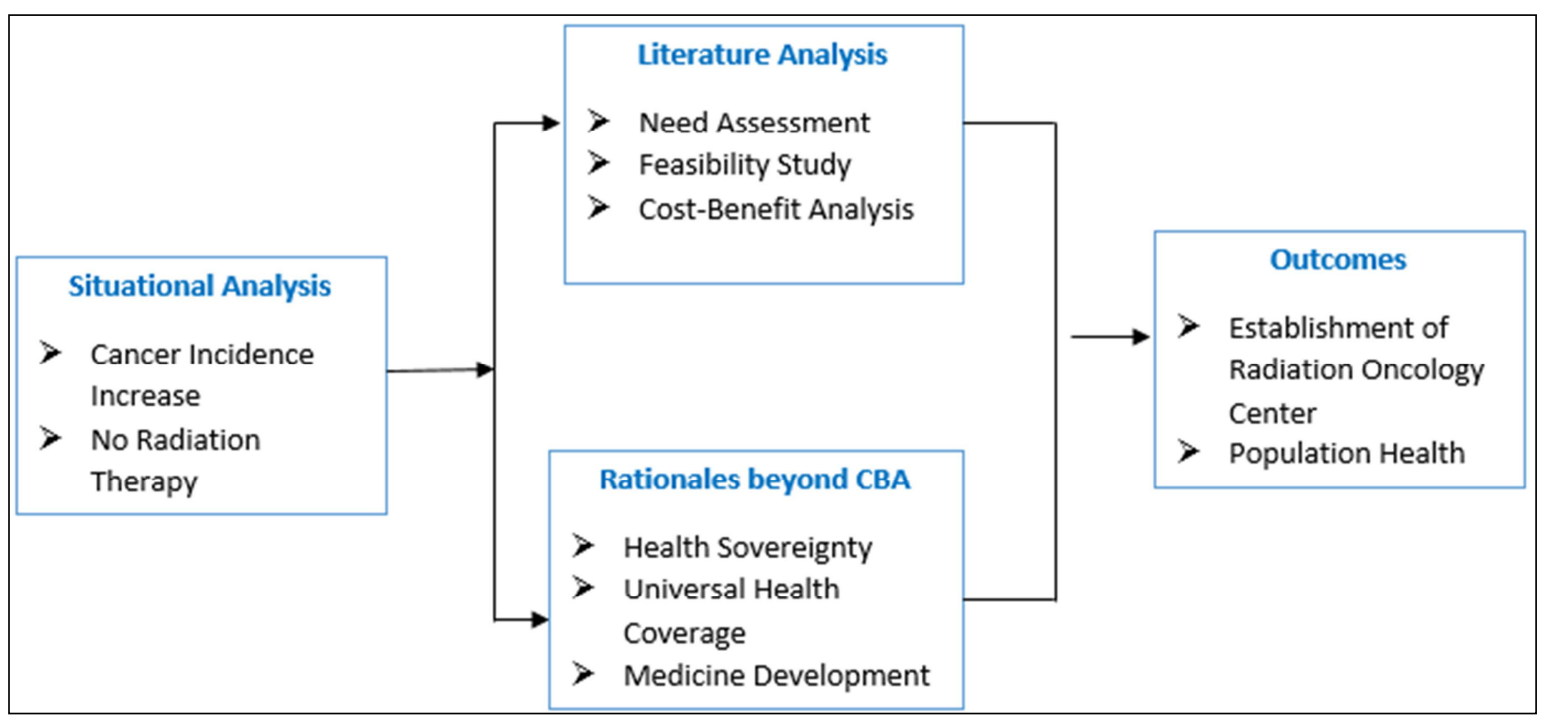

Figure 1. Research conceptual model on radiation oncology center in Fiji.

\section{The Current Situation on Cancer Treatment and Oncology Center}

\subsection{Cancer Treatments in Fiji}

In Fiji, basic surgery, limited chemotherapy and hormone therapy for cancer diagnosis and treatment are provided at the tertiary care level. The limited oncology services are provided by surgeons, physicians and pediatricians.
Radiotherapy is an essential component in treatment of cancer patients and some tumors, such as advanced cervical cancer, can only be cured by radiotherapy [2]. Nevertheless, due to the absence of radiotherapy services, patients are referred to neighboring countries on a cost share basis. For the three-year period between 2012 and 2014, 57 patients were treated on this basis, at a cost (in government subsidies) of 1,087,474 USD [4]. Overall, according to a government report on cancer treatment, the situation in Fiji is not good: 
"Late presentation with locally advanced or metastatic diseases is the norm. Anecdotally, approximately $80-90 \%$ of patients are diagnosed with incurable cancer. The vast majority receives no cancer treatment and relies on supportive care and morphine. In the case of cervical cancer, for example, there are an estimated 200 new cases per annum, of which approximately 25 patients receive 'curative' surgery and an additional 25 patients are referred for radiation treatment abroad. The remaining 150 are provided with supportive care but no cancer therapy" [2]

To deal with this challenging situation, the Government released the National Cancer Control Plan (NCCP) in 2014, which contained the roadmap for cancer control activities in Fiji. At the same time, the government established a National Oncology Committee (NOC) to work with experts working both in clinical and policy areas [6].

\subsection{Research on Radiotherapy Centre}

In February 2014 the Fiji government endorsed a plan to establish a radiotherapy center. The Cabinet approved the plan in principle, pending investigation of the financing and human resources required [7]. Following this, a series of research exercises were conducted by well-known expert groups and organizations. In March 2014, a Needs Assessment was undertaken by the International Atomic Energy Agency (IAEA), the World Health Organization (WHO) and the International Agency for Research on Cancer (IARC). This research concluded that based on the estimated cancer incidence and cancer patterns in Fiji there is a need for radiotherapy services, including brachytherapy, adding that the demand may be higher if the radiotherapy treatment facility were to extend its services to patients from neighboring Pacific Island countries [4].

Based on this Needs Assessment, the Government of Fiji requested a Feasibility Study on the establishment of a radiation oncology center. In June 2015, the study team led by the IAEA was launched, consisting of a medical physicist, radiation oncologist, architect, and medical imaging specialist [3]. The Feasibility Study covers initial construction of a radiation oncology facility with potential provision for subsequent expansion to a comprehensive cancer center in the future. Recommendations on initial equipment, staffing and associated costs were given, along with estimates for training, employment and recurrent costs that would be needed for the sustainability of the center. The study summed up with conclusion that "the project is considered to be feasible due to the commitment of the Fiji Government to its establishment and recognition of the resource requirements for development and sustainability" $[3]$.

In February 2016, a Cost and Benefit Analysis (CBA) was conducted by a team from Seoul National University led by Professor Soonman Kwon. The study compared the costs and benefits of establishing radiation oncology services in Fiji from a societal perspective, which took into account all the relevant costs and benefits. The time span for the CBA was 15 years -from 2021 to 2035 - due to the 15-year lifespan of radiotherapy equipment. According to the results of the study, the total benefit of establishing a radiotherapy center is estimated to be $59,052,381 \mathrm{FJD}$, while the expected cost for the center is $63,915,741$ FJD. The benefit estimation included: cost savings from avoiding overseas treatment $(17,626,666$ FJD) and life-years gained from unmet needs $(41,425,715$ FJD). The cost estimation was calculated on the basis of initial costs for construction and equipment (21,330,746 FJD) and operational costs, such as employment of healthcare professionals and maintenance $(42,584,995$ FJD). Based on these calculations, the expected net benefit was $-4,836,360$ FJD and thus the cost-benefit ratio was 0.92 , slight shy of 1.0 , which translates to the conclusion that establishing and operating a radiation center in Fiji was not cost beneficial [8] [9].

Table 1. Expected net benefit from cost-benefit analysis (unit: FJD).

\begin{tabular}{lll}
\hline Total cost & Total benefit & Net benefit \\
\hline $63,915,741$ & $59,052,381$ & B/C ratio \\
$\cdot$ Initial cost: & Cost savings from overseas treatment: & \\
$21,330,746$ & $17,626,666$ & $-4,863,360$ \\
$\cdot$ Operational cost: & Life-years gained from unmet needs: \\
$42,584,995$ & $41,425,715$ & 0.92 \\
\hline
\end{tabular}

Source: Kwon. S. et al (2016)

As the researchers mentioned in the study, there are caveats that should be considered, including benefits that were not covered in their study. First, the study did not consider the benefit to be gained from providing cancer treatments to other Pacific Island Countries and Territories (PICTs). Fiji in fact has established Memorandum of Understanding with seven Pacific Small Islands Developing States. These specifically tailored MOUs between Fiji and the Pacific countries are focused on the area of health and pharmaceuticals. They can contribute to the development of a radiation oncology center for cancer by using economies of scale and making Fiji a health care hub [2]. Second, the study did not include the benefit of the cost savings that would be made from reducing the longer-term cancer treatment that would need to be given to patients who did not receive proper radiotherapy. Third, benefits derived from delivering palliative care was not considered, given that radiotherapy is commonly used to reduce pain for terminal cancer patients [8] [9]. Considering these additional benefits that were not included in the cost-benefit study, the CBR (0.92) could be reversed to become cost-beneficial.

In May 2016, MoHMS and IAEA published the Bankable Document (BD) that comprehensively outlined a plan for implementing radiotherapy services for cancer patients in Fiji 
and described the necessary components needed to enable this to happen [2]. This report was written by combining all previous research results and thus was the final and most comprehensive report on the establishment of radiation oncology services in Fiji. The document discussed the main reasons why Fiji needs a radiation oncology center such as equity and accessibility, population health, and the benefits of becoming a medical hub in the South Pacific region. The Document mentioned three key findings;

Firstly, a very small number of cancer patients requiring radiotherapy get the opportunity to be referred to India, New Zealand or Australia, even on cost share basis (Table 1) [10]. Furthermore, the government subsidy covers medical costs only, resulting in very large out-of-pocket (OOP) spending by patients. Therefore, even if a patient is selected as a beneficiary of overseas treatment, he/she still needs to pay other costs such as airfares and accommodation, which frequently results in patient having to abandon the possibility of such treatment [2].

Table 2. Cancer patients treated overseas with Government subsidies 2012-2014.

\begin{tabular}{llll}
\hline Year & No. of patients & Name of hospital & Treatment cost (FJD) \\
\hline 2012 & 13 & & Airfare (FJD) \\
& 3 & Batra, India & $\$ 240,524$ \\
& 7 & Sahyadri, India & $\$ 34,560$ \\
& 3 & Shanti Mukand, India & $\$ 167,222$ \\
2013 & 17 & & $\$ 38,703$ \\
& 13 & Apollo/Sahyadri, India & $\$ 406,237$ \\
& 4 & Shanti Mukand, India & $\$ 284,882$ \\
2014 & 27 & & $\$ 121,354$ \\
& 5 & Batra, India & $\$ 440,713$ \\
& 18 & Sahyadri, India & $\$ 78,516$ \\
& 3 & Shanti Mukand, India & $\$ 282,629$ \\
& 1 & St George Hos, New Zealand & $\$ 79,568$ \\
\hline
\end{tabular}

Source: Ministry of Health and Medical Services (2015).

Secondly, a radiation center is needed to improve the quality of life of cancer patients. In the current system governing access to overseas treatment, the health and welfare of patients can deteriorate: the selection procedure may take about four weeks - and not being treated in a timely manner can lead to negative health outcomes for patients. In addition, the selection of candidates for overseas treatments focuses on patients who are more likely to be cured. Overall, patients with metastatic cancer with less chance for remission through radiotherapy treatment are less likely to be overseas treatment candidates, even though radiation therapy can be used to mitigate pain for patients at the terminal stage of their cancer.

Thirdly, an oncology center in Fiji could become a cancer treatment hub for all South Pacific countries, not only for Fijians. Countries located in this region, in general, are so small that it is difficult to realize economies of scale for radiation services. To achieve cost-effectiveness in establishing an oncology center, cooperation and collective operation - through combining small countries - is a good policy option [11]. Implementing radiotherapy services in Fiji would be a way to provide access to essential cancer treatment for people from Pacific Island Countries and Territories (PICTs) who would normally not be able to access such services [2].

\subsection{Stakeholder Opinions}

In health policy processes stakeholders' opinions are important, because a new policy can influence them directly or indirectly. Regarding the establishment of a radiation oncology center in Fiji, the major stakeholders are the Fiji Medical Association, Fiji Cancer Society, and the Fijian government. Unlike other social policies in the country, there is no major objection to the establishment of such a center. So far, there have been no contrasting policies or other conflicts over the construction of a radiation therapy center [3].

The government is one of the key stakeholders in establishing the radiation therapy center in Fiji as it is responsible for financing health services on the one hand and for the provision of services to meet the health needs of the population on the other hand. The government has approved an over-arching oncology policy, which mandates the formation of an Oncology Committee. The Fiji Cancer Society, as an NGO, supports the policy to proposal of a radiation oncology center. The organization took part in several committees which discussed the establishment of the center. Through its participation in the committee, the Fiji Cancer Society expressed their ideas and suggested ways to improve cancer patients' health. According to the Bankable Document report [2], the Fiji Cancer Society underlines the importance of local treatment with support for accommodation and transport from the outer islands. Furthermore, the Society is expected to play a role in monitoring and providing designated accommodation for patients. The Fiji Medical Association has no reason to oppose a centre dedicated to providing radiation therapy as the association would be subject to less pressure from cancer patients if such a center were to open. Without an oncology center, patients would be more likely to complain because they tend to think that basic services, such as cancer 
counseling and Pap smear testing for suspected cancer patients, should be provided within the country's primary and secondary care facilities. Furthermore, tertiary hospitals would be able to refer cancer patients to the radiation treatment center after basic surgery, which would greatly improve patients' course of treatment.

\section{Rationales for a Radiation Oncology Center}

As highlighted in the previous section, many research papers and documents on the proposal to establish an oncology center have provided theoretical or empirical findings. While these documents have differed slightly in their approach, they all have reached in same conclusion that radiation therapy is urgently needed and necessary for cancer patients both in Fiji and in nearby small South Pacific countries. Apart from academic-based research, the major cancer-related stakeholders in Fiji have expressed their interest in and support for an oncology center to improve the health of cancer patients who currently do not have timely or any access to radiation treatment.

Given the situation, what should be done next? In line with the opinions of many researchers and experts, it is time to pass the baton to the government. The government's willingness to press ahead is critically important for the implementation of the oncology project in Fiji. The implementation plan should be completed and operationalized. An Action Plan can be prepared based on existing research results and experts' documents which outline a number of ideas as well as sample roadmaps for establishing the center. In order to implement an action plan confidently, central government needs a simple but clear rationale to accelerate the project effectively. The following rationales can be guidelines in support of decision-makers within the Fijian government.

\subsection{Health Sovereignty}

Health sovereignty is generally considered to be the exercise of a state's sovereign power to protect health and provide health services [12]. Based on the idea of health sovereignty, scholars have promoted the principle of 'health as an inalienable right' ie. that governments should provide health services to their populations. Gibson (1988) strongly views the delivery of health care as a right to which all in need should have equal access [13]. Jones (1983) perceives "disease as one of the threats to the well-being of the population" and thinks that citizens "should be provided protection from major threats to life. The primary function of the state is to protect citizens" [14].
Based on this principle, governments in modern welfare states have obligations to provide health services to all people living in their territories. Furthermore, rather than waiting for the government to provide health care, citizens first request the state to do something for their health [15] and the government consequently should ensure people's safety and health by guaranteeing basic health services and medicines. In fact, in terms of health sovereignty there are several examples where governments are already implementing this principle globally. According to the Implementation of Paragraph 6 of the Doha Declaration on the TRIPs Agreement and Public Health, governments can use the so called "Compulsory License" to protect people's life. Under the compulsory license, governments can manufacture pharmaceuticals regardless of patent rights. Moreover, governments use the principle of health sovereignty as leverage to protect people' right to health when there is a need to protect peoples' medical information by prohibiting its delivery or transfer to other countries. In addition, to secure and to implement health sovereignty, developed countries, as well as some developing countries, exempt cancer patients from out-of-pocket (OOP) payments or impose lower levels of cost-sharing for these patients, because catastrophic diseases like cancers and hospitalization are not elastic to price and thus will not likely lead to moral hazard. Granting exemptions from OOP payments for cancer treatment is a global trend. In Fiji however, currently the opposite occurs: rather than reducing the financial burden of cancer patients, such patients in Fiji are faced with high costsharing when they use radiation therapy services in foreign countries.

\subsection{Universal Health Coverage (UHC)}

In general UHC means "all people can access the health services they need without incurring financial hardship" [16]. In the health sector, UHC is an urgent and critically important issue, especially in developing countries [17]. Recognizing this, in 2005 the Member States of the World Health Organization (WHO) adopted a resolution encouraging countries to develop health financing systems aimed at providing universal health coverage. As the cubic dimensions in $<$ Figure $2>$ show, UHC consists of three key components: the extent of population coverage, the extent of health services covered (and their quality), and protection from suffering financial hardship through excessive costsharing [18] [19]. Based on the important goal of UHC, the United Nations recently endorsed UHC as the third Sustainable Development Goals (SDGs) in order to ensure people's protection from catastrophic health expenditure and access to essential health services [20]. 


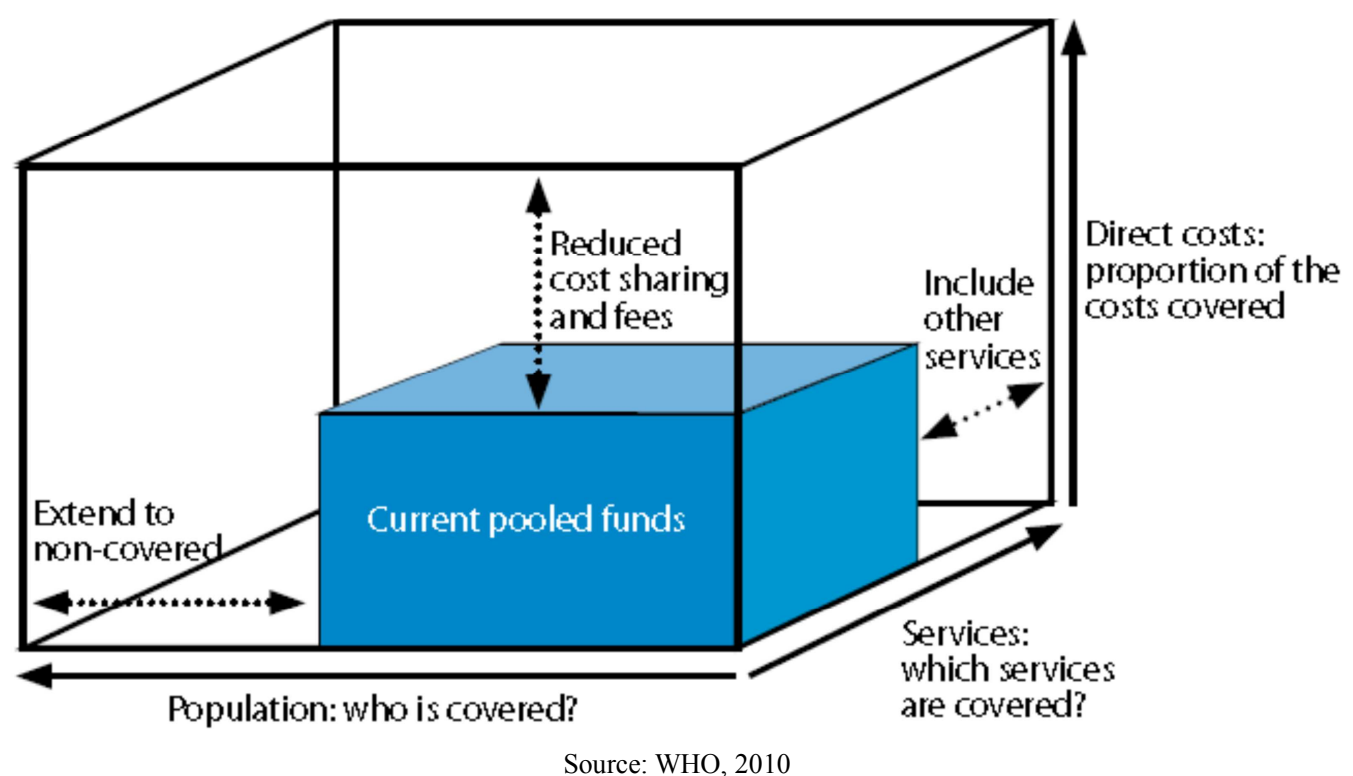

Figure 2. Three dimensions towards Universal Health Coverage.

Looking in particular at cancer treatment in Fiji from the perspective of these three dimensions of UHC, it is clear that much work needs to be done to improve the situation [21]. In terms of population coverage, very few cancer patients have access to radiation therapy because few patients can obtain entitlement to treatment abroad. Only a small percentage of cancer patients are able to obtain treatment if they pass the selection criteria set by the authorization committee. The absence of nationally-provided radiation therapy highlights one of the limitations to the benefits package (extent of services covered). Generally, the provision of cancer services in Fiji are somewhat fragmented compared to other health services under the country's strong publicly financed health care system, which delivers outpatient and inpatient services free of charge. Finally, the financial burden placed on those patients who do qualify for radiation therapy abroad is substantial given the cost-sharing that is required for the treatment itself, in addition to patients having to cover airfares and accommodation for themselves and any accompanying family members. It is clear, therefore, that from the perspective of guaranteeing UHC, Fiji needs to ensure much greater equity in accessing cancer treatment, which will also enhance social solidarity [22]. It is not an overstatement to say that UHC in Fiji will not be possible without creating access to cancer treatment services.

\subsection{A Cancer Treatment Hub in the South Pacific Region}

Fiji's population is the largest in the South Pacific region which consists of several small countries and territories. Fiji, in terms of geopolitical and country size, is the hub of South Pacific, such as aviation and pharmaceuticals, and is host to many international organizations, including Asian Development Bank, the Pacific Islands Forum Secretariat and the World Health Organization Regional Office for the Western Pacific. In addition, Fiji already has established MOUs with seven Pacific Small Islands Developing States (PSIDS) for the development of health and pharmaceuticals [23]. Other countries in the South Pacific are too small to achieve economies of scale in their health sectors. For example, Palau (with a population of 19,700) has established and maintains chemotherapy services for cancer treatment but sends patients who need radiotherapy to overseas countries [24]. However, looking at the small island country of the Republic of Mauritius, with a small population of 1.3 million, may be instructive for Fiji. Mauritius has established one radiotherapy center, and it has the potential to be further developed to provide comprehensive oncology treatment for cancer patients [8] [9]. These examples might lead Fiji to consider the option of becoming a cancer treatment hub by establishing and operating a radiation therapy center for the populations of both Fiji and other neighboring countries. Considering the expected increase in cancer incidence across the South Pacific region (see $<$ Table $3>$ ), a radiation therapy center in Fiji has the potential to become a hub for cancer treatment services in this region.

Table 3. The number of cancer incidence in South Pacific region.

\begin{tabular}{llll}
\hline Region & 5 most common Cancers (both sexes) & New cancer cases in 2015 & Projected cancer cases in 2025 \\
\hline Melanesia & Breast, Cervix, Prostate, Lip and oral cavity and liver & 10,992 & 14,773 \\
Micronesia & Prostate, Breast, Lung, Colorectal, Corpus uteri & 860 & 1,201 \\
Polynesia & Prostate, Breast, Lung, Corpus uteri, Cervix & 1,332 & 1,733 \\
Total & & 13,184 & 17,707 \\
\hline
\end{tabular}

Source: MoHMS and IAEA (2016). 
In addition, establishing a radiation therapy center can lead to the development of medical practice in the country by upgrading the capacity of professionals in the health and health-related industrial sectors. Radiation oncologists, radiation therapy technologists, and other specialists in radiation therapy area can play an important role in the development of medicine in Fiji [25]. Modern radiotherapy and particularly radiation equipment, like linear accelerators (linacs), is increasingly improving the quality of treatment through the delivery of complex treatment techniques and imaging using automated computer controlled hardware [6]. A radiation center would also introduce nuclear medicine to Fiji. Using nuclear medicine imaging technology, such as PET imaging, is considered to be an indispensable imaging technique in the modern management of cancer patients. Last but not least, the highly sophisticated technology that would be used in the radiation oncology center could lead to the development of other health-related industries that would contribute to the health improvement of Fijians and people from many neighboring countries.

\section{Conclusion}

With changes in lifestyle and eating habits, the incidence of NCDs, led by cancers, will increase rapidly both in developed and developing countries. Thus, many countries are focusing their attention on NCD-related diseases. For example, in the United States the age standardized mortality rate from cancers in 2015 was 158.6 per 100,000 people. In South Korea, the mortality rate from cancers was 150.8 in 2015 , which means that approximately $28 \%$ of people (one in four) is dying of cancer. The situation in Fiji is not an exception. NCDs are responsible for $80 \%$ of all deaths in Fiji. According to IARC GLOBOCAN 2012 data, the age standardized mortality rate is $65.4 / 100,000$ (239 cases) in men and 104.3/100,000 (418 cases) in women. In 2015 the numbers of new cases are estimated at 1,194 . These numbers are predicted to increase to 1,437 in 2025 and 1,619 in 2034.

As income increases, Fijians will increase their interest in obtaining good quality healthcare gradually. As a result, more cancer patients will want to go abroad to receive cancer treatment. This will lead to rising demand for the government to fund overseas treatments, and thus to the need for higher budgets. The economic and health expenditure burden of cancers in Fiji will rapidly increase in the future. In line with the increase in demand, the expectations to establish a radiation oncology center will also increase, not only among researchers who have argued in favour of it but also in light of the endorsement already given by Fiji's government. With the establishment of a radiotherapy center, cancer patients in Fiji as well as other South Pacific Countries would have access to timely radiation therapy.

Many previous studies have concluded that Fiji needs a radiation therapy center and the government has reviewed these research findings thoroughly over a long period of time. Moreover, there is already national consensus regarding the establishment of a radiation therapy center. Cancer patients in Fiji do not want to travel overseas to receive treatment by paying high costs out-of-pocket. Likewise, patients living in neighboring South Pacific countries do not want to travel long distances to obtain radiation treatment in India or Australia. In addition to these policy considerations, there is a strong argument to be made that an oncology center in Fiji could be the cornerstone to securing health sovereignty and would contribute to achieving universal health coverage (UHC). It also has the potential to enhance economic development by making Fiji a medical hub in the region. For its successful implementation, the Fijian government will need to exercise good stewardship for the people of Fiji and also for neighboring populations.

\section{Conflict of Interest}

There are no conflicts of interest to report.

\section{Acknowledgements}

The author would like to acknowledge the feedback from members of the National Oncology Committee in Fiji and Dr. Luisa Cikanarana for their comments. Also, special thanks to Anna Maresso, Manjulai Lal, and Shivika Bhandhana for valuable feedback.

\section{References}

[1] Ministry of Health and Medical Services (2015). Annual Report 2014. Suva: 2015. Available from: http://www.health.gov.fj/?page id=198.

[2] Ministry of Health and Medical Services and International Atomic Energy Agency (2016), Improving the treatment outcomes and quality of lives for cancer patients in Fiji and neighboring 'Small Island Nations' through the implementation of radiotherapy services in Suva, Fiji. Bankable Document.

[3] International Atomic Energy Agency. (2015). IAEA Mission Fiji (Project No: RAS007031).

[4] International Atomic Energy Agency (IAEA), the World Health Organization (WHO) and the International Agency for Research on Cancer (IARC). (2014). Integrated Missions of PACT (imPACT) Report: Cancer Control Capacity and Needs Assessment Report.

[5] Ministry of Health and Medical Services. (2016). National Strategic Plan 2016-2020. Suva.

[6] Ministry of Health and Medical Services (2017). National Cancer Control Strategic Plan (2017-2021), Suva.

[7] Office of the Prime Minister (2014). Memorandum: Cabinet Decision No. 23 on the subject of Radio Oncology Centre. Suva.

[8] Eunkyoung K. Yoon-min, Cho, Soonman K, Kunhee Park (2017). Cost-benefit analysis of establishing and operating radiation oncology services in Fiji. Cancer Epidemiology. 50 (2017) 247-256. 
[9] Kwon, S. Park, S. M, Kim, E., Cho, Y. M., (2016). CostBenefit Analysis in Establishing and Operating Radiation Oncology Services in Fiji. Seoul.

[10] Ministry of Health and Medical Services (2015). Cancer patients treated overseas with government subsidies. Suva, Republic of Fiji.

[11] Government of Fiji and IAEA (2015). Country Programme Framework 2016-2020. Suva.

[12] Werkhseiser, I. (2014). Food Sovereignty, Health Sovereignty, and self-organized community viability. Interdisciplinary Environmental Review. Vol. 15, Nos. 2/3.

[13] Gibson, R. D. (1988). We the people: Our Inalienable Right to Quality Health Care. American Pharmacy No. 28 (12): 19-21. December.

[14] Jones, Gary (1983). The Right to Health Care and the State. Philosophical Quarterly. 33 (132):279-287. July.

[15] Van der Vyver, J. D. (1989). The Right to Medical Care. Medicine and Law 7 (6):579-583.

[16] Carrin G. Mathauer, I Xu, K and Evans D. (2008). Universal Coverage of Health Services: Tailoring Its Implementation. Bulletin of the World Health Organization. November 2008. 86 (11).

[17] Russo G, Bloom, G, and McCoy D. (2017). Universal health coverage, economics slowdown and system resilience: Africa's policy dilemma.
[18] Agyepong et al. (2016). The "Universal" in the UHC and Ghana's National Health Insurance Scheme: Policy and Implementation Challenges and Dilemmas of a Lower Middle Income Country. BMC Health Services Research. (2016) 16:504 DOI 10.1186/s12913-016-1758-y.

[19] World Health Organization (2010). Health Systems Financing: The Path to Universal Coverage. Geneva.

[20] Tangcharoensathien, V., Mills, A., Palu, T. (2015). Accelerating Health Equity: the Key Role of Universal Health Coverage in the Sustainable Development Goals. BMC Med. 13 (1). 101.

[21] MoHMS. (2018). Universal Health Coverage. NHEC Memorrandum. Suva, Rewa Province, Fiji: MoHMS.

[22] World Health Organization (2014). Strategy for Universal Health Coverage. $154^{\text {th }}$ session of the executive committee. WHO regional office for the America. Washington.

[23] MoHMS. (2018). International Agency for Research on Cancer Pacific Islands Hub for Cancer Registry. NHEC Memorandum. Suva, Rewa, Fiji: MoHMS.

[24] Palau Ministry of Health (2006). National Cancer Strategic Plan for Palau: 2007-2012.

[25] International Atomic Energy Agency (2008). Setting up a Radiotherapy Program: Clinical, Medical Physics, Radiation Protection and Safety Aspects. IAEA. 